\title{
Broken Symmetries in Spacetime with Torsion and Galactic Magnetic Fields without Dynamo Amplification
}

\author{
Luis Carlos Garcia de Andrade \\ Department of Theoretical Physics, IF-UERJ, Rio de Janeiro, Maracanã \\ Email: garcia@dft.if.uerj.br
}

Received February 6, 2012; revised March 15, 2012; accepted March 26, 2012

\begin{abstract}
Since Kostelecky et al. [Physical Review Letters 100, 111102 (2008)], have shown that there is an intimate connection between spacetime with torsion and the possibility of constraining it to Lorentz violation, a renewed interest in torsion theories of gravity has arised. In this paper, minimal coupling between photons on a torsioned background is shown to allow us to obtain the galactic magnetic field strength $\mu G$ without dynamo amplification. This agrees with recent results by Jimenez and Maroto (2011) for spiral galaxies, with galactic magnetic field constraints from Dark matter without dynamo amplification. The approach discussed here allows us to get rid of the unpleasant photon mass by simply considering the Lagrangean cut off for second order torsion terms. Therefore though the gauge and Lorentz symmetries are broken here one does not have to deal with photon masses.
\end{abstract}

Keywords: Star Dynamos; Torsion Theories; Lorentz-Violation; Cosmology; Astro-Particle Physics

\section{Introduction}

There are many papers where galactic magnetic fields [1] and not only primordial fields can be obtained from a Biermann battery type and not from a usual dynamo mechanism of magnetic field amplification. More recently Jimenez and Maroto cite [2] have shown that in the presence of dark energy, magnetic fields are generated without any amplification. They obtained for spiral galaxies fields of the strength of $10^{-9} \mathrm{G}$ in accordance with Schuster-Blackett conjecture that the magnetic fields are obtained from angular momentum. Earlier on De Sabbata and Gasperini [3] showed that this conjecture could be placed in the realm of Einstein-Cartan gravity [4], where spin of the elementary particles are the Cartan torsion source [5]. More recent Opher and Wichoski [6] have shown that the same mechanism can be used to obtain galactic magnetic fields without dynamos amplification. Also Kostelecky et al. [7] investigated the Lorentz violation (LV) of the fermionic sector of a Lagrangean, in Riemann-Cartan spacetime the curvature tensor $R_{i j k l}$ and the e.m sector $F^{i j} F^{k l},(i, j=0,1,2,3)$ given by $R_{i j k l} F^{i j} F^{k l}$ displays the same symmetries of LV term, where the Riemann-Cartan curvature tensor, including torsion terms plays the role of the Higgs sector constants $k_{i j k l}$. The main difference from previous papers [8] is that one uses here a minimal coupling instead the non-minimal photon-torsion coupling where the magnetic vector potential does not interact directly with torsion or contortion part in the QED Lagrangean. In this paper, we show that the use of this photon sector coupled with torsion flat mode, via non-minimal coupling yields naturally the breaking of gauge fields and a strength for the galactic magnetic fields of the observed $\mu G$ value without the dynamo amplification. Besides the advantage of the approach here is that by considering only first order terms on torsion, one is able to avoid the uncomfortable photon tiny mass. The paper is organised as follows : In Section 2 we consider the Riemann-Cartan (RC) sector of the Lagrangean obtain a 2D system of Maxwell generalised equation which are similar to the ones obtained for the neutrino mass oscillation. Section 5 contains conclusions and discussions.

\section{Flat Photon-Torsion Minimal Coupling and Magnetic Field Amplification}

Since the torsion effects are cosmological weak in the galaxy formation post inflationary era, compared to curvature effects of Einstein gravity sector, second-order torsion effects are neglected in the torsioned quantum electrodynamics (QED). Photon and gravity torsion sectors are given by [9]

$$
\begin{aligned}
S= & \frac{1}{m^{2}} \int \mathrm{d}^{4} x(-g)^{\frac{1}{2}}\left(-\frac{1}{4} F^{i j} F_{i j}+a R^{*} F^{i j} F_{i j}\right. \\
& \left.+b R_{i k}^{*} F^{i l} F_{l}^{k}+c R_{i j k l}^{*} F^{i j} F^{k l}+d D_{i} F^{i j} D_{k} F_{j}^{k}\right)
\end{aligned}
$$


The physical constants $a, b, c, d$ are obtained by means of the conventional Feynman diagram techniques [10]. Here the e.m field tensor $F_{i j}$ are given by the minimal coupling through torsion gravity by the covariant derivative

$$
\nabla^{i} A_{j}=\partial_{i} A_{j}-K_{i j k} A^{k}
$$

where $K$ represents the contortion part of the RiemannCartan connection. By using this expression for the covariant derivative of the magnetic potential vector in spacetime, in the expression for the Maxwell sector of the Lagrangean one obtains

$$
F_{i k}^{*} F^{* i k}=F^{2}-2 A_{k} \varepsilon^{k l m n} K_{n} F^{l m}
$$

where $F^{*}$ comes from the two-differential form of the e.m field coupled minimally to torsion as [9]

$$
F_{i j}^{*}=F_{i j}-2 A^{k} \varepsilon_{k i j n} K_{n}
$$

where $K_{i}$ is the Cartan torsion vector and $\varepsilon_{k i j n}$ is the totally skew-symmetric Levi-Civita symbol. The magnetic field tensor non-miminally coupled would be $F^{i j}=\partial^{i} A^{j}-\partial^{j} A^{i}$. To simplify matters, only the axial part of the contortion tensor $K_{i j k}$ in the form

$$
K^{i}=\varepsilon^{i j k l} K_{j k l}
$$

Since the term $R_{i j k l} F^{* i j} F^{* k l}$ contains second-order terms in torsion they reduce to the usual term $R_{i j k l} F^{i j} F^{k l}$. Of course this is possible since we are using Minkowski metric which does not allow us to consider post-inflationary models. The Maxwell generalised equations in Fourier space reduce to

$$
\partial_{i} A^{i}=\nabla \times \boldsymbol{A}=i \boldsymbol{k} \times \boldsymbol{A}=0
$$

where $\boldsymbol{k}$ is the wave vector chosen here as $\boldsymbol{k}=(0, k, 0)$. With this choice the remaining Maxwell like equations read

$$
\begin{aligned}
& \left(\partial_{\eta}^{2}-k^{2}\right) A^{1}-i\left[\varepsilon^{2} k^{2}+2\right] k^{2} K^{0} A^{3}=0 \\
& \left(\partial_{\eta}^{2}-k^{2}\right) A^{3}-i\left[\varepsilon^{2} k^{2}+2\right] k^{2} K^{0} A^{1}=0
\end{aligned}
$$

where $\eta$ is the conformal time given by the Minkowskian metric $\mathrm{d} s^{2}=\mathrm{d} \eta^{2}-\mathrm{d} x^{2}$. Here the ansatz for the magnetic vector potential is $A=A_{0} e^{i \omega \eta}$ and $K^{0}$ is the only contortion component different from zero. Here also, $A^{0}$ and $A^{2}$ components vanish. The frequency $\omega$ is to be determined by the determinant of the Jacobian $\mathrm{J}$ part of the matrix obtained from the last two equations

$$
\left(\begin{array}{cc}
\left(\omega^{2}-k^{2}\right) & \left(-i\left(\varepsilon^{2} k^{2}+2\right) k^{2} K^{0}\right) \\
\left(i\left(\varepsilon^{2} k^{2}+2\right) k^{2} K^{0}\right) & \left(\omega^{2}-k^{2}\right)
\end{array}\right)
$$

Thus by considering the two dimensional column matrix $A^{T}=\left(A^{1}, A^{3}\right)$ one may write the Maxwell generalised equation as

$$
J A=0
$$

Therefore the equation $\operatorname{Det} J=0$ allows us to obtain the dispersion relation for the photon-torsion spacetime background as

$$
\omega=k\left[1 \pm\left(1+\frac{\varepsilon^{2} k^{2}}{2}\right) K^{0}\right]
$$

Now to compute the components of the magnetic field in terms of the vector electromagnetic potential one simply use the expression

$$
\boldsymbol{B}=\nabla \times \boldsymbol{A}=i \boldsymbol{k} \times \boldsymbol{A}
$$

Which yields

$$
\begin{aligned}
& B^{3}=i k^{2} A^{1} \\
& B^{1}=i k^{2} A^{3}
\end{aligned}
$$

Taking now the square root of these expressions as our magnetic field one obtains the following expression

$$
B \approx 10^{11} K^{0}
$$

on a coherent scale of $100 \mathrm{Mpc}$ one obtains $B \approx 10^{-6} \mathrm{G}$ which is the observed galactic magnetic field. This is obtained for a torsion field compatible to the one obtained from Hughes-Drever experiment estimates [11] as $K^{0} \approx 10^{-17} \mathrm{~m}^{-1}$.

\section{Discussion and Conclusion}

It is shown here that by considering only first order effects on spacetime torsion, and photon-torsion minimal coupling the photon mass introduced by the gauge symmetry breaking does not appear in the Maxwell generalised equations which allows us to obtain the galactic magnetic field without dynamo amplification. On a future publication one could address the problem of investigating post-inflationary models primordial field generation by considering the extension of the equations here to include a Friedmann cosmology background.

\section{Acknowledgements}

I would like to express my gratitude to D. Sokoloff and R. Soldati for helpful discussions on the subject of this paper. Financial support from CNPq. and University of State of Rio de Janeiro (UERJ) are grateful acknowledged.

\section{REFERENCES}

[1] A. M. Howard and R. Kulsrud, "The Evolution of a Primordial Galactic Magnetic Field,” The Astrophysical Journal, Vol. 483, No. 2, 1997, p. 483. doi:10.1086/304262

[2] J. B. Jimenez and A. L. Maroto, “On the Electromagnetic 
Nature of Dark Energy and the Origin of Cosmic Magnetic Fields," Progress of Theoretical Physics Supplement, in Press.

[3] V. de Sabbata and M. Gasperini, "Gauge Invariance, SemiMinimal Coupling, and Propagating Torsion,” Physical Review D, Vol. 23, 1982, p. 2116.

[4] V. de Sabbata and C. Sivaram, "Spin and Torsion in Gravitation,” World Scientific, Singapore, 1995.

[5] L. C. G. de Andrade and C. Sivaram, "Torsion, Massive Electrodynamics and Primordial Magnetic Fields in the Early Universe," Astrophysics and Space Science, Vol. 209, No. 1, 1993, pp. 109-111. doi:10.1007/BF00666441

[6] R. Opher and U. F. Wichoski, “Origin of Magnetic fields in the Universe and due to Non-Minimal Coupling of Gravitational and Electromagnetic Coupling,” Physical Review Letters, Vol. 78, No. 5, 1997, p. 787. doi:10.1103/PhysRevLett.78.787

[7] A Kostelecky, N. Russell and J. Tasson, "Constraints on Torsion from Lorents Violation,” Physical Review Letters,
Vol. 100, 2008, Article ID: 111102.

[8] L. C. G. de Andrade, "Galactic Dynamos from Torsion Flat Modes and Lorents Violation,” Physical Letters B, 2011.

[9] L. C. G. de Andrade, "Constraints on Torsion from the Bosonic Sector of Lorents Violation and Magnetogenesis Data,” Nuclear Physics B, Vol. 847, No. 3, 2011, p. 485.

[10] I. T. Drummond and S. J. Hathrell, "QED Vacuum Polarisation in Background Gravitational Field and Its Influence in the Velocity of Photon," Physical Review D, Vol. 22, No. 2, 1980, p. 343. doi:10.1103/PhysRevD.22.343

[11] B. R. Heckel, E. G. Adelberger, C. E. Cramer, T. S. Cook, S. Schlamminger and U. Schmidt, "Preffered Frame and CP Violation Tests with Polarised Electrons," Physical Review D, Vol. 78, 2008, Article ID: 092006. doi:10.1103/PhysRevD.78.092006 